\title{
Electron Backscatter Diffraction Analysis of Single Pass Builds of Additively Manufactured Inconel 625
}

\author{
N. D. Hart ${ }^{1}$, B.W. Baker ${ }^{1}$, T.T. Roehling ${ }^{2}$, M.J. Matthews ${ }^{3}$ \\ ${ }^{1}$ Mechanical Engineering Department, United States Naval Academy, Annapolis, MD, USA \\ ${ }^{2}$ Department of Mechanical Engineering, University of the Pacific, Stockton, CA, USA \\ ${ }^{3}$ Materials Science Division, Lawrence Livermore National Laboratory, Livermore, CA, USA
}

Many current metal additive manufacturing (AM) systems employ powder bed fusion processes. Thin layers of powder are applied to a substrate, and an energy source such as a laser is used to fuse the powder into specific geometries, building a part layer-by-layer [1]. In this investigation, single laser-melted weld tracks of Inconel 625 are analyzed by electron backscatter diffraction (EBSD) to determine the heat effect of and grain morphology produced by laser powder bed fusion (LPBG). Previous research without EBSD has been reported on 316L stainless steel using the same experimental setup [2]. Depending on alloy composition and cooling conditions, rapid solidification can produce a wide variety of microstructures [3]. This variation leads to difficulty in modeling and predicting material properties. The purpose of this research is to determine if EBSD can be used to better understand grain morphology and ultimately assist in identifying processing parameters to improve the overall AM process.

In this work Inconel 625 powder was consolidated onto an Inconel 718 substrates using a range of laser powers, scan speeds, and energy densities (Table 1). The experimental scheme was discussed in a previous report [2]. The laser tracks were cross-sectioned and mounted in conductive resin. A final polishing step of $0.05 \mu \mathrm{m}$ colloidal silica on a vibratory polisher was used without etching to prepare the samples for analysis. EBSD was conducted using a TESCAN MIRA3 Field Emission Gun microscope at $20 \mathrm{keV}$ using an EDAX Digiview camera system. Imaging was performed at $10 \mathrm{keV}$ using a probe current of approximately $1 \mathrm{nA}$ on the same as-polished samples.

Backscattered electron (BSE) imaging showed the development of a laser nugget consisting of a bead above the substrate surface, a root area corresponding to melt penetration into the substrate, and an overall melt zone (Fig. 1). EBSD analysis of the laser nugget was achievable and showed a columnar grain structure correlating to the build direction. Inverse pole figure maps of melt tracks produced under different processing conditions are shown in Fig. 2. Of the tracks studied (Table 1), all processing conditions produced long columnar grains emanating from the substrate through the laser nugget in the build direction. In addition to EBSD, energy dispersive spectroscopy (results not reported here) was conducted to determine if particles formed in the laser nugget. Unlike many Inconel alloys where $\mathrm{Ti}, \mathrm{Nb}$, or $\mathrm{Ta}$ particles form during normal manufacture, no particles were observed in the laser nugget.

The results clearly show that EBSD of single-pass AM structures is possible and that AM produces columnar grain structures with grains aligned in the build direction and with no observable formation of particles. Follow on research will determine if subsequent heat treatment can recrystallize the AM grain structure. Initial results on whole AM parts have shown that similar Inconel 625 builds do not recrystallize during post-AM processing. 


\section{References:}

[1] W. King, A. Anderson, R. Ferencz, N. Hodge, C. Kamath, S. Khairallah, and A. Rubenchik, Applied Physics Reviews, 2015, vol. 2, p. 041304.

[2] T.T. Roehling, S.S.Q. Wu, S.A. Khairallah, J.D. Roehling, S.S. Soezeri, M.F. Crumb, and M.J. Matthews, Acta Mater., 2017, vol. 128, pp. 197-206.

[3] W.J. Boettinger, Mater. Sci. Eng., 1988, vol. 98, pp. 123-130.

Table 1. Laser parameters used.

\begin{tabular}{|c|c|c|c|}
\hline Track & Power $(\mathrm{W})$ & Speed $(\mathrm{m} / \mathrm{s})$ & Energy Density $\left(\mathrm{J} / \mathrm{mm}^{3}\right)$ \\
\hline 1 & 50 & 0.125 & 80 \\
\hline 2 & 150 & 0.375 & 80 \\
\hline 3 & 250 & 0.625 & 80 \\
\hline 4 & 350 & 0.875 & 80 \\
\hline 5 & 450 & 1.125 & 80 \\
\hline 6 & 550 & 1.375 & 80 \\
\hline
\end{tabular}
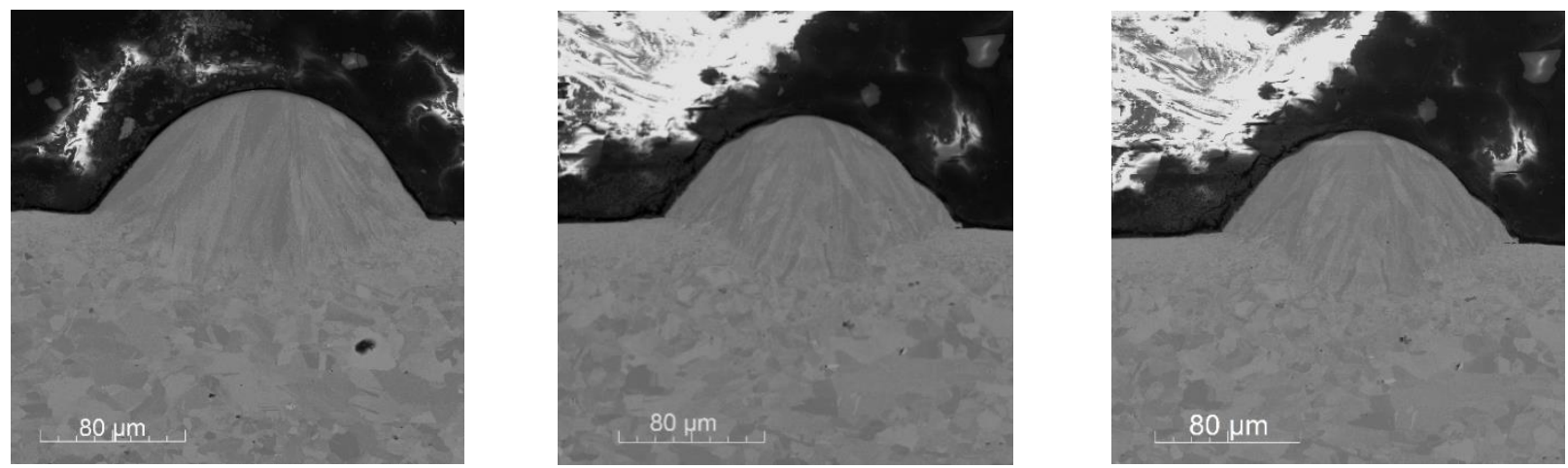

Figure 1. Backscatterd electron imagery of typical laser nuggets formed during single pass builds of INCONEL 625 powder on a INCONEL 718 substrate. Images are from Tracks 3, 4, and left to right showing a range of laser power and speeds but identical energy densities. All iamges are 250 micron sqaure view vields with scale bars as shown.
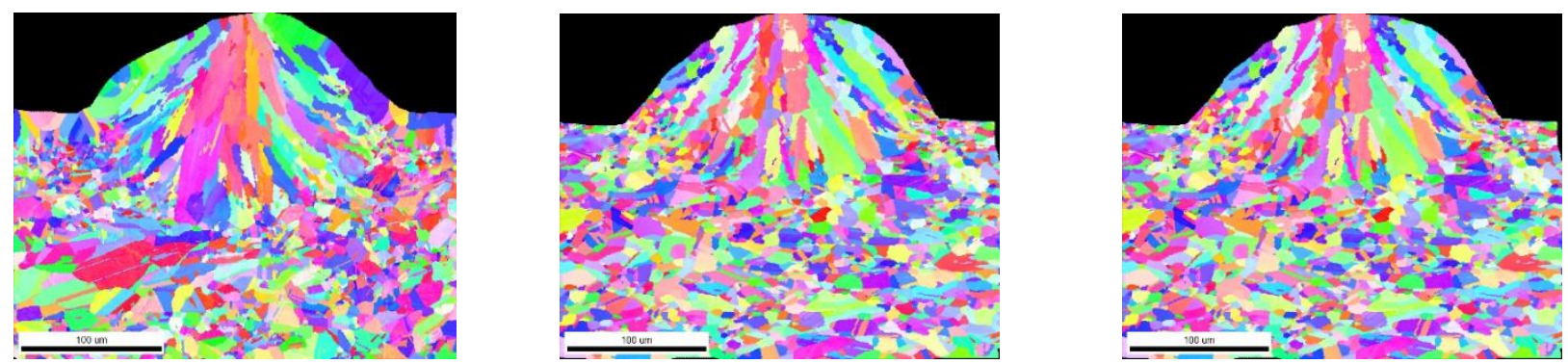

Figure 2. Inverse pole figure maps of the laser nugget and substrate. Images correspond to tracks 3, 4, and 6 similar to Fig. 1 .

\section{Acknowledgements:}

This work was funded through a Laboratory Directed Research and Development grant 15-ERD037 and performed under the auspices of the U.S. Department of Energy by Lawrence Livermore National Laboratory under contract DE-AC52-07NA27344. 